\title{
SUR LA FILAMENTISATION DES MONILIA
}

\author{
Par R.-V. TALICE
}

Les Monilia (1), dont la morphologie et la biologie sont si particulières, constituent un groupe de champignons très importants en pathologie humaine et animale par la fréquence de leur parasitisme, la variété de leurs localisations dans l'organisme et les conséquences morbides qui en résultent. Malheureusement et malgré l'intérêt que présentent surtout les Monilia parasites de l'appareil respiratoire et du tube digestif, une seule espèce a été étudiée à fond au point de vue botanique: Monilia albicans (Ch. Robin, 1853), agent du muguet; le nombre des travaux publiés sur ce champignon est actuellement incalculable. Pour cette raison, et avant d'exposer la question du développement des Monilia en général, nous croyons nécessaire de donner un bref aperçu historique sur le $M$. albicans.

\section{Historique}

L'histoire étiologique du muguet, maladie connue dès la plus haute antiquité, comporte les étapes principales suivantes :

$1^{\circ}$ En 1839, Langenbeck, le premier, reconnaît que la maladie est liée à l'existence d'un champignon.

$2^{\circ}$ En 1842, Berg, de Stockholm, découvre le parasite du muguet, donne quelques détails sur sa morphologie et réussit à le cultiver en cultures impures.

$3^{\circ}$ En 1847, Ch. Robin, étudiant les lésions chez l'homme, donne, avec planches à l'appui, une bonne description du parasite, qu'il nomma en 1853 Oidium albicans.

$4^{\circ}$ A partir de 1877 , Grawitz cherche à le cultiver à l'état pur sur divers milieux, complète la description de Ch. Robin en distinguant la forme levure et la forme filamenteuse, mais identifie par erreur l'Oidium albicans au Mycoderma vini.

$5^{\circ}$ En 1885, Stumpf prétend dissocier le champignon en deux espèces distinctes : l'une se présentant exclusivement sous la forme levure, l'autre sous la forme filamenteuse.

(1) Nous comprenons sous ce nom les champignons de la famille Torulopsidacer Ciferri groupés dans les genres Candida, Mycotorula, Enantiothammus, voir Crferri (P.) et Redaelli (P.). - Studies on the Torulopsidaceae. Annales Mycologici, XXYII, 1929, p. 243-295.

AnNales de Parasitologie, T. VIII, $\mathrm{N}^{\text {os }} 3-4 .-1^{\text {er }}$ juillet 1930 , p. 394-410. 
$6^{\circ}$ En 1883, Plaut applique à l'étude du champignon les procédés bactériologiques modernes et l'identifie au Monilia candida de Bonorden.

$8^{\circ}$ En 1890, Roux et Linossier démontrent que certains organes de ce Monilia, mal interprétés par les auteurs précédents, étaient en réalité des chlamydospores. Ils étudient aussi les conditions de développement de chacune des deux formes végétatives du champignon.

$9^{\circ}$ La question de la pluralité étiologique de la maladie avait déjà été posée par divers auteurs (Rajat et Péju, Montpellier et Catanei), mais ce sont surtout Castellani et Redaelli qui démontrent que d'autres champignons, en dehors de $M$. allicans (Ch. Robin, 1853), sont capables de produire le syndrome clinique de muguet. Nous nous rangeons entièrement à cette opinion, mais il est vrai aussi que ce parasite cosmopolite si répandu est celui qu'on trouve présent dans la grande majorité des cas de la maladie.

\section{Dimorphisme Des Monilia}

Donc, depuis longtemps, on a reconnu que le $M$. albicans est un champignon dimorphe qui peut se présenter, soit dans les lésions, soit dans les cultures, sous deux formes différentes: une forme globuleuse ou blastosporée ou levure et une forme filamenteuse improprement appelée aussi mycélienne. Ces deux formes, toutes deux végétatives, sont reliées par de nombreux intermédiaires; mais pour la commodité de la description, on choisit les types extrêmes. Cette particularité du $M$. albicans, commune d'ailleurs à tous les Monilia parasites, a été beaucoup étudiée et a donné lieu à des interprétations très différentes.

Il est important de connaître les facteurs qui déterminent ce dimorphisme, non seulement au point de vue biologique général, mais aussi pour la caractérisation du genre et pour la systématique des espèces, systématique basée jusqu'ici sur des données très aléatoires telles que les réactions biologiques.

La morphologie des blastosporés qui poussent exclusivement sous la forme levure est trop uniforme et trop simple pour permettre la détermination des espèces en se basant sur ce seul caractère. Il y a donc tout intérêt à observer et à étudier comparativement les Monilia, surtout sous la forme filamenteuse, plus riche en détails morphologiques, d'autant plus qu'on ne connaît pas encore chez ces champignons d'organes reproducteurs nettement définis (1). Il

(1) Nous laissons naturellement de côté le genre Endomyces, qui présente des asques et auquel appartient une forme de Monilia albicans qui est l'Endomyces vuillemini Landrieu, 1912. 
faut, par conséquent, les cultiver dans des milieux offrant les meilleures conditions pour le développement des filaments.

Ce sont précisément les essais que nous sommes en train de faire avec le $\mathrm{D}^{\mathrm{r}}$ Langeron qui nous ont conduit à une revision critique et expérimentale de tous les facteurs qu'on a supposés en cause dans la filamentisation. Voici les faits historiques principaux qui concernent cette question :

Berg, en 1842, dans les cultures faites à la température ambiante sur un milieu azoté acide et aussi dans une solution de sucre de canne avec ou sans blanc d'œuf, n'avait constaté que la forme levure ou blastosporée du champignon. Le même fait fut constaté par Grawitz sur les milieux suivants : $1^{\circ}$ solution de sucre de raisin additionnée d'acide tartrique et de 2 p. 100 de sels minéraux (cendres de cigares) ; $2^{\circ}$ décoction de prunes.

Ch. Robin (1853) décrit la forme filamenteuse dans les lésions parasitaires. Grawitz trouve les deux formes dans les cultures impures. Kehrer (1883) observe les filaments dans les cultures sur décoctions de prunes et eonclut de ses expériences que la présence de la gomme arabique empêche leur apparition.

Ensuite les auteurs s'attachent à l'étude des conditions qui favorisent quantitativement le développement de chacune des deux formes de croissance du champignon. Plaut démontre que la forme filamenteuse apparaît surtout dans les cultures mises à des températures élevées. En 1877, Fischl résume ainsi ses observations : les milieux pauvres en sucre, pauvres en amidon, privés d'azote, les milieux liquides et l'anaérobiose favorisent la filamentisation ; tandis que l'addition de sucre, la richesse en amidon, les milieux solides et l'aérobiose favorisent la croissance sous la forme levure. Plaut, Stumpf observent que les filaments se présentent abondamment dans les cultures sur petit lait. Audry (1887) démontre le peu d'importance de la réaction du milieu sur le phénomène et écrit : "Ce n'est pas sur le bouillon que nous avons pu observer le développement mycélien le plus abondant... C'est sur le vin stérilisé. Là seulement, nous avons pu voir des touffes de mycélium considérables arrangées en tourbillons presque opaques... "

Plaut, Heller (1885), Kraus (1926), Moro (1910) constatent l'influence favorable du sérum sanguin sur la filamentisation. Roux et Linossier (1890) considèrent surtout les facteurs chimiques: dans les milieux glycosés ils ne trouvent que des formes levures, les formes filamenteuses prédominent, au contraire, sur les milieux albuminoïdes purs. Ils ont ensuite établi les rapports des deux formes de végétation dans une série de milieux naturels :

Milieux liquides: forme globulo-filamenteuse dans le jus de 
tomate, la décoction de carottes, le bouillon de Lœffler, le vin chauffé, le lait, le bouillon de touraillons et le moût de bière ; forme levure dans l'urine, le vin non chauffé et le moût de vin.

Milieux solides : forme globulo-filamenteuse sur le melon, la gélatine saccharosée ; forme levure, au début, sur la carotte, la pomme de terre cuite, la cerise, la fraise ; forme levure exclusive sur la tomate crue, la rave, la betterave cuite, le concombre cru, le citron cru, l'abricot, la courge cuite, le marron d'Inde cuit, la gélatine peptonée, la gélatine aux touraillons et la figue fraîche crue.

En outre, une action curieuse a été signalée par ces auteurs : l'addition d'une faible quantité de nitrate d'ammoniaque à un milieu liquide amènerait rapidement la formation d'un grand nombre de filaments. Comme on retrouve le sel intact dans le milieu, " son action semblerait être purement catalytique ". Ils affirment aussi, contrairement à Kehrer, que l'addition de la gomme arabique aux cultures favorise la production des filaments.

En 1890, Teissier, contrairement à Audry, obtient des résultats très différents selon la réaction du milieu : "Sur gélose acide, tant que la réaction restait la même, on trouvait exclusivement la forme levure ; dès que la culture devenait alcaline, des filaments courts, puis longs, apparaissaient... Le passage sur gélose alcaline du champignon cultivé sur gélose acide (même après des ensemencements en série sur ce milieu) donne la forme filamenteuse..., le passage sur gélose acide du champignon semé en série sur gélose alcaline donne la forme levure exclusive... La réaction acide d'un milieu solide favorise donc le développement de la forme levure et la réaction alcaline la forme filamenteuse."

Dans ces dernières années, les auteurs allemands ont étudié soigneusement l'influence des facteurs physico-chimiques sur la filamentisation du "Soorpilz ". Hahn et Junker (1928) concluent que la tension superficielle a une influence très nette sur le dimorphisme du champignon; l'abondance de la filamentisation serait en raison directe de l'intensité de la tension superficielle des milieux. Les expériences ont été faites avec une grande quantité de substances ajoutées à un liquide type (maltose 5, nitrate d'ammoniaque 0,01 , eau de conduite 100 ). Les milieux les plus favorables à la filamentisation sont donc ceux qui sont constitués par des substances à tension superficielle élevée : vin rouge, décoction de prunes, petit lait, sérum.

Milochevitch (1929) étudie le développement quantitatif et qualitatif de $M$. albicans, surtout sur des milieux à tension superficielle connue, préparés avec des extraits d'organes humains et conclut comme Hahn et Junker. Ainsi il n'observa pas de filaments dans le 
liquide de Sauton (tens. sup. 0,44 p. 100), très peu dans le liquide de Raulin (tens. sup. 1,17 p. 100), une assez grande quantité dans l'urine (tens. sup. 17,85 p. 100) et le bouillon (tens. sup. 18 p. 100), plus encore dans l'extrait de sous-maxillaire-bouillon (tens. sup. 40,47 p. 100) et l'extrait de poumon-bouillon (tens. sup. 40,47 p. 100) (1).

\section{Facteurs QUi déterminent LE DimorPhisme}

De l'ensemble de toutes ces recherches, il résulte que les conclusions des divers auteurs sont très différentes les unes des autres et très souvent contradictoires. Nous en donnons quelques exemples démonstratifs : la réaction du milieu, d'après Plaut, n'a aucune influence sur la filamentisation, tandis que pour Teissier elle règle le mode de développement du champignon. L'addition de gomme arabique aux cultures inhibe la formation de filaments d'après Kehrer, la favorise d'après Roux et Linossier. La décoction de prunes ne donne que la forme levure pour Grawitz, tandis que pour Kehrer, Hahn et Junker, etc., le même milieu donne aussi la forme filamenteuse. Dans le vin chauffé, Roux et Linossier observent rarement la forme globulo-filamenteuse, contrairement à Audry, Hahn et Junker. La richesse en azote des milieux a une influence favorable sur la filamentisation pour Plaut, défavorable pour Fischl. L'urine donne exclusivement la forme levure pour Roux et Linossier; pour Milochevich le même milieu donne " assez de filaments ". Audry n'observe que la forme levure sur les milieux solides, contrairement à beaucoup d'auteurs. On remarquera également la tendance à considérer un seul facteur déterminant et à exclure les autres.

Pour tâcher d'expliquer, au moins en partie, ces contradictions si profondes, il faut d'abord se demander quelle espèce de champignon a été étudiée par les divers auteurs. Malgré l'importance de ce point, dans beaucoup de travaux, on parle simplement du " champignon du muguet " (auteurs français) et du "Soorpilz " (auteurs allemands) sans qu'il soit question d'une description morphologique et d'une détermination préalables du germe. La plupart des auteurs ont admis que le muguet est une maladie causée par une seule espèce de champignon ; pourtant, actuellement, on paraît admettre que plusieurs espèces sont en cause. En

(1) Ces chiffres, obtenus avec le stalagmomètre de Traube, représentent le nombre de gouttes du liquide en question excédant celui de la même quantité d'eau à la même température et rapporté à 100 (pour plus de détails voir HAHN et JUNKER, Kolloidbiologische Studien über Soor. Jahrb. $f$. Kinderheilkunde, CXXI, 1918, p. 85-100). 
outre, beaucoup de chercheurs, même modernes, se sont contentés d'expérimenter avec une seule souche de Monilia. Déjà, les quelques auteurs (Noisette, Daïreuva) qui en ont étudié plus d'une ont pu constater des différences entre elles. Dans un prochain mémoire nous démontrerons à quels résultats invraisemblables on peut arriver quand on prétend déterminer des champignons comme les Monilia par des réactions biologiques sans se préoccuper préalablement de leurs caractères morphologiques. Le manque fréquent d'une description microscopique du Monilia en culture pure et même l'absence de contrôle d'ordre biologique pour s'assurer de la détermination exacte de l'espèce, expliquent, à notre avis, une grande partie de ces contradictions. Finalement, même si l'on veut admettre que dans toutes ces recherches il s'agit toujours du $M$. albicans, ce que nous savons de la variabilité in vitro de ces champignons, variabilité que nos recherches personnelles nous ont également permis de confirmer, nous oblige à être très prudents dans les conclusions générales tirées de l'étude d'une seule souche de Monilia.

Nous analyserons maintenant séparément les facteurs qui ont été signalés comme déterminant la filamentisation. En même temps nous indiquerons les résultats de nos recherches, faites avec 30 souches de Monilia de différente provenance, appartenant à plusieurs espèces isolées de l'organisme de l'homme et du hérisson, chez des sujets sains ou malades. Les souches du hérisson proviennent d'un matériel que le Prof. Brumpt a bien voulu nous donner à étudier.

\section{Influence de la consistance des milieux}

Milieux solides. - Les auteurs sont presque unanimes à déclarer que le "champignon du muguet " ne forme que des éléments globulaires sur les milieux solides. L'absence des filaments est mentionnée sur des tranches de citron par Quinquaud (1868), sur le pain (Baginsky, 1885), sur la pomme de terre (Baginsky, Noisette, 1875), sur gélatine (Grawitz, 1868 ; Grasset, 1893 ; Noisette), sur la betterave (Linossier et Roux, 1690), sur gélose (Grasset, Noisette). Audry avait insisté spécialement sur la constance absolue de la forme levure, à tout âge, sur tous les milieux solides. A la même opinion se rangent Fischl, Plaut, Teissier, Hahn et Junker, etc.

Mais, depuis longtemps, on avait remarqué que les choses se passent souvent différemment. Baginsky (1885) observe, dans la gélatine inoculée par piqûre que les filaments apparaissent dans la 
profondeur du milieu, dans les parties éloignées de la surface ou de la ligne d'inoculation. Roux et Linossier (1890) signalent des formes globulo-filamenteuses, au début, sur quelques milieux solides : melon, gélatine saccharosée. Teissier observe des filaments dans la gélose alcaline. Laurent (1891) obtient de très belles cultures filamenteuses sur le moût de bière gélatiné. Noisette (1898) affirme que dans les cultures impures (avec staphylocoques par exemple), il se forme des filaments qui pénètrent dans les parties liquéfiées par les microbes. Les auteurs allemands ont trouvé des filaments sur le sérum coagulé.

Mlle Daïreuva, en 1899, écrit : "Partout et toujours nous avons trouvé des filaments, aussi bien sur les organismes vivants que dans les cultures sur les milieux les plus variés, même sur ceux qu'on avait considérés jusqu'ici comme nourrissant exclusivement la forme levure..., les globules sont en plus grand nombre à la surface des plaques crémeuses développées dans la bouche et sur les milieux de culture solides, et, à un examen superficiel, on peut ne pas trouver d'autres éléments... "Elle a également signalé la capacité que présente le champignon d'émettre sur les cultures en milieux solides naturels ou artificiels des filaments qui pénètrent dans la profondeur du milieu. Milochevitch (1929) signale les fila* ments sur la gélose-foie, la gélose-cerveau et la gélose-glande sousmaxillaire, préparées avec des organes humains.

Nous avons voulu élucider cette question en cultivant plusieurs souches de Monilia sur : gélose amidon soluble à 4 p. 100 ; gélose amidon soluble à 4 p. 100 , peptonée à 1 p. 100 ; gélose dextrinée à 4 p. 100 ; gélose dextrinée à 4 p. 100 , peptonée à 1 p. 100 ; gélose dextrinée à 4 p. 100 , peptonée à 1 p. 100 , additionnée de 0,5 p. 100 de chlorure de sodium; gélose amidon soluble à $4 \mathrm{p}$. 100, peptonée à 1 p. 100 , additionnée de 0,5 p. 100 de chlorure de sodium ; gélose amidon soluble à 4 p. 100 , additionnée de 0,50 p. 100 de chlorure de sodium (1); gélose peptonée à 1 p. 100 ; colle de farine de blé, sérum coagulé, milieu de conservation de Sabouraud, milieu d'épreuve glycosé de Sabouraud à 2 p. 100,4 p. 100 et 6 p. 100 , milieu de Gorodkowa, carotte. La température de culture a été toujours de $37^{\circ}$. On a commencé avec une souche de Monilia (genre Candida) qui donnait aisément de filaments sur les milieux liquides. Voici les résultats de ces expériences :

Monilia 255 (souche Ashford : Monilia tropicalis) ; culture mère sur milieu glycosé de Sabouraud à 2 p. 100.

(1) Les milieux qui précèdent ont été établis par Langeron et Milochevitch pour leurs recherches sur les dermatophytes publiées dans ces Annales (VIII, 1930, p. $422-436)$. 
Au bout de 36 heures : très peu de filaments sur sérum coagulé, gélose amidon soluble et gélose-peptone; beaucoup sur les autres milieux. Au bout de 72 heures, les blastospores prenaient le dessus dans tous les milieux; au bout de 120 heures on observe peu de filaments dans tous les tubes. La filamentisation normale est surtout abondante sur gélose dextrinée peptonée et sur gélose amidonnée peptonée. Sur milieu glycosé de Sabouraud à $6 \mathrm{p}$. 100, la culture est très abondante, mais le champignon végète sous un aspect filamentoïde anormal. Par filaments nous n'entendons pas, comme certains auteurs, des articles plus ou moins allongés, placés bout à bout, mais de tubes longs, cylindriques, ramifiés, cloisonnés, donnant des blastospores latérales ou terminales.

Nous avons ensuite cultivé un Monilia qui avait presque perdu la propriété de donner des filaments à la suite d'un grand nombre de passages sur divers milieux solides.

Monilia 11 (isolé de la bouche d'un enfant sain, à Paris) ; culture mère sur milieu glycosé de Sabouraud à 2 p. 100 .

Au bout de 36 heures, les tubes qui montrent le développement le plus abondant sont ceux du milieu de Sabouraud glycosé à 6 p. 100 et de gélose dextrine-peptone, mais c'est seulement ce dernier milieu qui montre la forme filamenteuse et celle-ci en grande abondance; au bout de 60 heures, la culture montrait exclusivement la forme levure.

Finalement, nous rapportons ici les observations très intéressantes que nous avons pu faire dans les cultures géantes de nos 30 souches de Monilia. Quand les colonies atteignent de grandes dimensions, par exemple au bout d'un mois ( 6 à 7 cent. de diam.), si on les examine par transparence, on constate dans presque toutes, soit à partir du centre, soit à partir de la périphérie, une quantité de stries radiées qui plongent dans l'épaisseur du milieu. Si l'on examine avec le binoculaire ces rayons qui forment comme un deuxième disque étoilé au-dessous du disque crémeux et lisse superficiel, on voit alors que chaque rayon est constitué par un axe filamenteux ramifié, quelquefois très long, entouré de véritables manchons de blastospores. Quelques auteurs ont observé le même phénomène en fixant en masse les cultures sur milieux solides et en les traitant comme des pièces histologiques.

En résumé : A. Sur les milieux solides les Monilia produisent des filaments dans les premières heures (24-48). Mais ensuite la croissance du champignon se fait sous la forme levure.

B. Les filaments s'observent seulement au contact de la surface du milieu. Mais, postérieurement, on peut observer, surtout quand on cultive le champignon dans de grands récipients, que les fila-

Annales de Parasitologie, $\mathrm{T}$. VIII, $\mathrm{N}^{\mathrm{os}} 3-4 .-1^{\mathrm{er}}$ juillet 1930.

26. 
ments plongent dans l'épaisseur du milieu et qu'ils se recouvrent rapidement de véritables manchons de blastospores.

C. Ici, comme ailleurs, on constate que les différentes souches se comportent de façon diverse et qu'une même souche donnera d'autant plus facilement des filaments qu'elle est plus habituée à végéter sous cette forme. On pourrait dans ce cas employer le mot de race.

Les souches ou races à filamentisation difficile ou disparue donnent difficilement la forme filamenteuse sur les milieux solides; seuls, les milieux dextrinés-peptonés ou fortement glycosés se sont montrés capables, dans une expérience, de déterminer une période très courte de filamentisation.

D. La filamentisation se produit dans les milieux solides purement albuminoïdes ou purement hydrocarbonés, mais surtout dans les milieux qui contiennent en même temps ces deux types de substances. Dans des expériences comparatives, les filaments normaux ont apparu en plus grand nombre sur les milieux dextrinéspeptonés.

E. La température optima est toujours de $37^{\circ}$.

Milieux demi-solides ou demi-liquides. - Avant de parler des milieux liquides, nous devons dire quelques mots sur la question des Monilia cultivés dans des milieux plus ou moins sirupeux. Grawitz (1877) parle de l'absence de filaments sur la décoction de prunes dès qu'elle est épaissie jusqu'à consistance sirupeuse. Comme nous l'avons dit, les milieux rendus sirupeux par l'addition de gomme arabique favorisent la production de filaments d'après Roux et Linossier et l'inhibent d'après Kehrer.

Cependant, Roux et Linossier signalent aussi le fait suivant: "Dans la gélatine-peptone sucrée avec le sucre de canne, on trouve, au bout de plusieurs mois, et alors que le substratum est devenu sirupeux, de longs filaments grêles... "

- Redaelli et Ciferri ont conseillé les milieux demi-solides (gélosés à 0,1 p. 100) pour provoquer la filamentisation des champignons du groupe des Torulopsidaceæ. Pour étudier ce facteur, nous avons cultivé comparativement quelques souches de Monilia sur des milieux liquides (eau de pomme de terre, bouillon glycosé à 2 p. 100 ) et sur des milieux demi-liquides (eau de pomme de terre gélosée à 0,1 p. 100 et à 0,3 p. 100 ; bouillon glycosé à 2 p. 100 , gélosé à 0,1 p. 100).

En résumé : pour les Monilia qui donnent facilement des filaments, les milieux liquides se sont montrés supérieurs aux milieux demi-liquides. Pour tous les Monilia qui donnent difficilement des 
filaments ou qui ne les donnent pas du tout, l'adjonction de gélose aux milieux liquides n'a aucun avantage.

Milieux liquides. -- Il est incontestable, et cela a été observé par presque tous les auteurs, que sur tous les milieux liquides les Monilia se développent sous la forme filamenteuse, au moins pendant les premiers jours.

Nous avons essayé une quantité de milieux liquides divers pour des cultures en tubes et en gouttes pendantes : eau de pomme de terre, eau de carotte, Raulin acide, bouillon glycosé à 2 p. 100 et à 4 p. 100 , solution aqueuse d'amidon soluble à 4 p. 100 , sérum animal, vin blanc, eau de pomme de terre peptonée à 1 p. 100 , solution aqueuse de dextrine à 4 p. 100 , peptone 1 p. 100 , solution aqueuse d'amidon à 4 p. 100 , peptone à 1 p. 100 , et d'autres dont nous parlerons à propos de l'influence des autres facteurs physiques et des facteurs chimiques. De tous les essais faits avec plusieurs souches de Monilia, nous concluons :

A. Qu'on choisisse, soit un Monilia à filamentisation facile, soit un Monilia à filamentisation difficile, la conclusion est toujours la même : le milieu le plus favorable pour la production des filaments et celui qui donne les résultats les plus constants, est l'eau de pomme de terre, milieu qui d'ailleurs nous avait été très vivement recommandé par notre maître, M. Langeron. Le sérum liquide semble aussi un bon milieu, mais l'observation de la colonie dans le Lugol par le procédé que nous conseillons plus loin, devient alors très difficile.

B. Les souches qui ont été cultivées longtemps sur des milieux solides et qui sont, par conséquent, habituées à végéter sous la forme blastosporée, donnent peu de filaments dans le premier ensemencement sur l'eau de pomme de terre, mais en donnent davantage dans les réensemencements successifs.

Dans des recherches faites avec le $\mathrm{D}^{r}$ Sautet sur le parasitisme des Monilia dans la bouche et dans l'intestin d'enfants sains ou malades des hôpitaux de Paris, nous avons constaté ce curieux phénomène : dans quelques cas, les exsudats examinés directement au microscope ne montrent que les formes levures d'un blastosporé qui croissait comme tel dans le premier ensemencement sur liquide de Raulin, dans le premier réensemencement et quelquefois jusqu'au deuxième et troisième passage sur le même milieu ou eau de pomme de terre. A partir de ce moment, on voyait apparaître les formes filamenteuses, seules capables de permettre l'identification du genre Monilia.

C. Pour obtenir un milieu encore meilleur, nous avons essayé de modifier la préparation de l'eau de pomme de terre. Nos recher- 
ches nous ont démontré que ce milieu non bouilli, ou bouilli, mais non chauffé à l'autoclave, ne permet que très difficilement la culture des Monilia. Donc le chauffage à $120^{\circ}$ provoque dans les constituants du liquide la transformation nécessaire pour les rendre aptes à la nutrition du champignon. Nous avons ajouté ensuite à ce milieu diverses substances (voir plus loin, à propos des facteurs chimiques), sans obtenir aucun avantage évident. Quant à la question de savoir quelle est la concentration la plus favorable, il nous semble, d'après nos recherches, que la meilleure est celle comprise entre 20 et 30 grammes de pulpe de pomme de terre râpée pour un litre d'eau.

\section{Influence de la réaction du milieu}

Roux et Linossier avaient recherché les effets de l'addition de certains acides ou de certaines bases sur les cultures du champignon du muguet. D'après eux, une acidité faible n'a aucune influence sur le dimorphisme; il y a seulement tendance à la filamentisation dès que la végétation se trouve entravée, l'excès d'acide agissant là comme une substance toxique, qui, même à dose n'arrêtant pas le développement du champignon, provoque l'apparition des filaments.

Teissier (1890) (loc. cit.) avait conclu que la réaction acide d'un milieu solide favorise le développement de la forme levure et la réaction alcaline celui de la forme filamenteuse. Au contraire, Hahn et Junker, Milochevitch pensent que la réaction du milieu n'a pas là une influence manifeste. Les résultats de nos expériences avec plusieurs souches de Monilia, cultivées comparativement sur divers milieux solides et liquides à $p \mathrm{H}$ différents, nous permettent d'affirmer aussi qu'on ne peut pas constater un rapport constant entre la réaction acide ou alcaline et l'apparition de la forme levure ou filamenteuse du Monilia. En effet, si on cultive une souche de Monilia habituée à pousser sous la forme blastosporée, l'alcalinite ou l'acidité du milieu ne s'est pas montrée capable de déterminer la production de filaments. Cependant, dans le cas des Monilia qui donnent facilement des filaments, ceux-ci s'observent en une plus grande abondance dans les tubes titrés à $p \mathrm{H} \mathrm{8,} \mathrm{c'est-à-dire} \mathrm{dans} \mathrm{les}$ milieux à réaction alcaline (Talice).

\section{Influence de I'anaérobiose}

Plaut a dit que la formation des filaments est favorisée par le manque d'air. Roux et Linossier pensent, au contraire, que l'excès 
d'oxygène exerce la même influence. En tous cas, le champignon du muguet in vitro est un aérobie strict. Charrin et Ostrowsky (1896) ont démontré que, dans les milieux privés d'oxygène, non seulement il ne s'accroît pas, mais il périt au bout de quelques jours ; les essais de réensemencement restent stériles. Nous avons pu faire la même constatation avec deux souches différentes cultivées en eau de pomme de terre sous une couche d'huile de vaseline.

Teissier pensait que les milieux liquides n'offraient pas aux Monilia albicans des conditions de végétabilité parfaite. Or, l'oxygène fait défaut dans les milieux liquides, où, effectivement, le champignon ne forme jamais de voile superficiel, comme le genre Mycoderma, et reste submergé " en donnant naissance à des filaments d'autant plus longs que la culture se fait au fond d'une couche de liquide plus épaisse " .

Nous avons cultivé quelques souches de Monilia dans des tubes contenant une colonne de liquide de hauteur variable et nous avons observé, en effet, que l'abondance de la filamentisation augmente proportionnellement avec la hauteur du liquide, mais au delà d'une certaine limite, elle est au contraire entravée. L'anaérobiose partielle est un facteur accessoire, mais non déterminant, parce que dans d'autres expériences comparatives, cette seule condition n'a pas pu provoquer la filamentisation dans les espèces à race blastosporée.

\section{Influence de la température}

Nous avons constaté, comme d'autres auteurs, qu'une température élevée augmente la production des filaments, sans cependant la déterminer. La température la plus favorable paraît être celle de $37^{\circ}$, tant pour les milieux solides que pour les milieux liquides. Nous considérons ce facteur comme un des plus importants.

\section{Influence de la tension superficielle}

Comme nous l'avons vu, certains auteurs (Hahn et Junker, Milochevitch, etc.) ont attribué une grande importance à ce facteur dans la filamentisation. Mais il ne faut pas oublier qu'ils ont expérimenté sur une seule souche de Monilia.

Nous ne pouvons pas nier que la tension superficielle puisse avoir quelque influence, mais notre longue expérience de la culture des Monilia nous permet de dire que ce n'est pas non plus un 
facteur déterminant de la filamentisation. Nous pouvons même signaler des résultats en contradiction avec ceux qui ont été relevés par les auteurs en question : le liquide de Raulin (tension superficielle très basse) nous a donné souvent d'abondants filaments dès les premiers ensemencements. L'addition de quantités variables de glycérine à l'eau de pomme de terre ne nous a pas donné de résultats favorables. En outre, si nous ne nous sommes pas attachés à obtenir des mesures absolues des liquides essayés, les mesures comparatives démontrent que ce ne sont pas les liquides à plus haute tension superficielle qui donnent la filamentisation la plus abondante. Finalement, on a bien vu que les milieux solides permettent aussi très souvent le développement de la forme filamenteuse.

Donc, dans les conditions pratiques, quand il s'agit d'obtenir in vitro une filamentisation rapide et abondante d'un Monilia, on peut procéder sans tenir compte de ce facteur dont l'influence, si elle existe, est évidemment secondaire.

\section{Influence de la composition chimique des milieux}

Cette influence est incontestable. Plaut avait déjà affirmé que les milieux pauvres en sucre, et pauvres en amidon favorisent la filamentisation. Mais ce sont surtout les expériences des auteurs français qui ont mis en relief l'importance des aliments dans le dimorphisme des Monilia. A la suite des essais de culture dans des milieux chimiques de composition variée, Roux et Linossier posent la loi suivante : "Dans les cultures de muguet, la complication de la forme croît avec le poids moléculaire de l'aliment. En d'autres termes, plus ce dernier est de structure compliquée, plus il y a tendance à la formation de filaments, plus ceux-ci s'allongent et deviennent grêles. "

Il est évident que les Monilia, comme la plupart des champignons, ont besoin pour vivre in vitro d'aliments protéiques et d'aliments hydrocarbonés. Mais, en dehors de ces conditions, la filamentisation paraît obéir à des facteurs plutôt quantitatifs que qualitatifs. Sans qu'on puisse nier que d'autres actions interviennent (peut-être ce que A. Sergent (1929) appelle "facteurs de croissance " des microbes), on peut affirmer qu'on observe les filaments, surtout dans les milieux liquides pauvres en substances hydrocarbonées et spécialement pauvres en substances protéiques.

Quant à l'influence franchement favorable qu'aurait, d'après Roux et Linossier, l'addition des sels d'ammoniaque, des expé- 
riences soignées, conduites avec trois souches cultivées en tubes et en cellules, ne nous ont pas permis de confirmer les résultats de ces auteurs.

\section{INTERPRÉTATION DU DIMORPHISME DES Monilia}

En parlant des faits historiques concernant les cultures de $M$. albicans, nous avons vu en même temps les diverses interprétations proposées pour expliquer leur dimorphisme. La forme levure serait le faciès végétatif normal du champignon pour quelques-uns, elle serait une forme accessoire pour d'autres. Mais, d'une façon générale, pour la plupart, ce dimorphisme résulte de l'influence du milieu sur le développement du champignon. Pour Stœcklin (1898), au contraire, l'âge de la culture l'emporte sur l'action du milieu ; les filaments représenteraient les formes initiales, les globules les formes adultes. L'influence des milieux se réduirait à accélérer ou à retarder l'apparition de la forme définitive. A l'appui de sa thèse, il invoque la présence de filaments sur les jeunes cultures et la prédominance des levures dans les anciennes. Nous croyons qu'on ne peut pas rejeter complètement cette interprétation, mais nous pensons aussi que l'apparition de la forme filamenteuse exige certaines conditions de vie précaire. Cela résulte de ce que nous avons dit plus haut : anaérobiose partielle, faible concentration d'aliments dans les milieux de culture.

Il faut encore insister sur la notion d'accoutumance ou de race dont nous avons déjà parlé : une souche, maintenue pendant plusieurs générations, grâce à des ensemencements successifs, même sur les milieux liquides, reprendra plus facilement dans les réensemencements la même forme que les souches qui n'ont pas reçu cette sorte d'accoutumance; nous pouvons donner l'exemple suivant : en cultivant tous les jours une souche de Monilia de la bouche, nous sommes arrivés à créer une race qui donne en goutte pendante à $25^{\circ}$, et mieux à $37^{\circ}$, sur eau de pomme de terre, une culture filamenteuse luxuriante, très riche en verticilles de blastospores, au bout de deux heures.

\section{Conclusions gÉnÉrales}

Le dimorphisme des Monilia est un phénomène très général dans ce groupe. Nous avons constaté ce dimorphisme dans 30 souches de provenances différentes, isolées de la bouche, de crachats, ou de selles de sujets sains ou malades, chez l'homme et chez le hérisson.

Ces champignons ont une tendance à adopter dans leur déve- 
loppement, surtout la forme levure ou blastosporée. L'accoutumance à ce mode de végétation peut créer une race de Monilia exclusivement ou presque exclusivement blastosporée.

La forme filamenteuse est surtout une forme jeune. On l'observe in vitro au commencement des cultures et sur presque tous les milieux. L'apparition des filaments suppose des conditions de vie précaire : anaérobiose partielle, milieux pauvres en éléments nutritifs, mais contenant des substances protéiques et hydrocarbonées. Les autres facteurs invoqués par les auteurs auraient pour nous une importance secondaire. Les milieux liquides sont les meilleurs, et parmi ces derniers l'eau de pomme de terre à 20-30 p. 1.000 nous a donné les résultats les plus constants et les plus favorables pour toutes les souches essayées.

La température optima pour la filamentisation des Monilia est de $37^{\circ}$; elle peut être quelquefois indispensable.

\section{DÉDUCTIONS PRATIQUES}

Les Monilia sont des parasites très fréquents des cavités naturelles de l'appareil digestif et des voies respiratoires de l'homme et certainement des animaux. Mais il faut toujours se rappeler qu'ils peuvent se présenter dans les exsudats seulement sous la forme levure (muguet sans filaments, etc.). Le diagnostic morphologique devra se baser sur les cultures en tubes et en cellules. Nous conseillons toujours l'isolement sur liquide de Raulin acide, qui est un milieu très défavorable au développement des bactéries. Mais la forme filamenteuse peut apparaître seulement après le premier, le deuxième et même le troisième passage. Pratiquement, un blastosporé qui, au troisième passage, ne donne pas de filaments, n'est pas un Monilia. Pour les cultures en eau de pomme de terre, nous conseillons des tubes de $16 \mathrm{~mm}$. de diamètre, garnis de ce liquide sur $5 \mathrm{~cm}$. de hauteur. Pour les cultures en cellules, il faut surtout employer l'eau de pomme de terre à $37^{\circ}$. Si l'on prend soin de mettre une couche très épaisse de lanoline et de placer les lames bien horizontalement sur un support dans l'étuve, la plupart des cultures se conservent bien pendant 48 heures. Il faut examiner souvent les cultures pour saisir le moment de l'optimum de filamentisation. Chaque souche a un optimum de temps variable. Si on abandonne les cellules dans l'étuve pour les examiner au bout de 48 heures, par exemple, on sera souvent surpris de ne trouver que des touffes de blastospores et des rares filaments.

Il faut quelquefois faire d'abord quelques passages de la souche dans l'eau de pomme de terre tous les jours ou tous les deux jours 
pour obtenir une race filamenteuse. Les cultures dans ce milieu présentent un autre avantage : elles peuvent être étudiées au microscope très commodément en employant la méthode suivante que nous conseillons très vivement et qui est une modification de la coloration au Lugol préconisée par Langeron : quańd les cultures sont arrivées au degré voulu de développement, on détache les lames sans les secouer pour ne pas faire tomber les blastospores des filaments, puis on les dépose face en dessous, sur un support approprié, dans l'étuve à $25^{\circ}$. Une fois la culture desséchée, on enlève comme d'ordinaire la lanoline et on dépose sur la colonie une goutte de Lugol fort, ou de Lugol faible si l'eau de pomme de terre est très riche en amidon, et on lute. L'examen de la préparation montre alors un aspect un peu analogue au fond noir : l'amidon est fortement coloré en bleu-noir, les champignons en acajou plus ou moins foncé, ce qui est très commode pour l'observation à de forts grossissements et surtout pour le dessin à la chambre claire. Malheureusement, ces préparations ne sont pas permanentes, mais elles se conservent assez bien pendant quelques mois.

\section{BiBLIOGRAPHIE}

Audry (Ch.). - Sur l'évolution du champignon du muguet. Rev. de Médecine, 1887. Berg (F.-Th.). - Soc. méd. suédoise, 1842 (cité par Hahn et Junker, 1928), - Über die Schwämmchen bei Kindern. Bremen, 1848 (cité par HahN et JUNKER).

Baginsky (A.). - Üeber Soorculturen. Deulsche med. Woch., 1885, p. 867 (cité par M. Dä̈reuva).

Charrin et Ostrovsky. - L'Oidium albicans, agent pathogène général. C. R. Soc. biol., Paris, 1896.

Castellani (A.). - Fungi and fungous diseases. Chicago, 1927, p. 107.

Daïreuva (M. P.). - Recherches sur le champignon du muguet et son pouvoir pathogène. Thèse de Nancy, $1899,86 \mathrm{p}$.

Fischl (R.). - Prager Med. Wochenschr., 1886, no 41. - Zentralbl. f. Kinderheil., I, 1887. - Fortschr. d. Med., V, 1887. - Erg. d. inn. Med. u. Kinderh., XVI, 1924 (cité par Hahn et Junker).

Grawitz (P.). - Virchow's Archiv., LXX, 1877; LXXIII, 1878, p. 147-150 ; CIII, 1886 (cité par Hahn et Junker).

Grasset. - Etude d'un champignon parasite de l'homme. Arch. de méd. expérim., 1893 (cité par Daïreuva).

Hahn (F.-V.) et Junker (H.). - Kolloidbiologische Studien über Soor. Jahrbuch. f. Kinderheilke, CXXI, 1928, p. 85-100.

Heller (A.). - Beitrag zur I.ehre vom Soor. Dtsch. Arch. f. ki. Med., LV, 1895 (cité par HaHN et Junker).

JUNKer (H.) et Wieben (M.). - Weitere Ergebnisse über die Wirkung extremer Potenzverdünnungen auf Organismen. Planta, II Teil, Versuche mit Pilzen. Die Mycelbildung von Soor, Planta, Arch. $f$. wissensch. Bolanik, VI, 1928, p. 473-481. 
Kraus (L.). - Cité par Rockstron (W.). - Neue Gesichtspunkte zur Myzelbildung des Soor und ihre Beziehung zur Therapie. Inaug. Dissert. Hamburg, 1926. Kehrer (F.-A.). - Über den Soorpilz. Heidelberg, 1883 (cité par Hahn et Junker). Langeron (M.). - Précis de Microscopie, 4e édit., Paris, Masson, 1925.

Launent (E.). - Bull. de la Soc. belge de Microscopie, XVI, 1890 (cité par P. Achalme. Le champignon du muguet. Gazette des Hôpitaux, LXIV, 1891).

Langenbeck (B.). - Frorieps's Notizen, 1839, n²52 (cité par Ch. RoBin).

MiLochevitch (S.). - Das Wachstum und die Wachstumsformen des Soorpilzes auf verschiedenen Nährböden. Zentralbl. $f$. Bakter. u. Infektionskrankheiten, CXIV, 1929, p. 174-185.

Moro (E.). - Soor dans Handb. $d$. Kinderh. von Pfaundler-Schlossmann., 2. Aufl., Leipzig, 1910, 3 Band (cité par HaHN et Junker).

Montpellier (S.) et Catanei (A.). - Présence du même Monilia chez deux malades porteurs de lésions différentes de la cavité buccale. C. R. Soc. Biol., LXXVIII, 1926, p. 568-569.

Norsetre (G.). - Recherches sur le champignon du muguet. Thèse de Paris, 1898, $193 \mathrm{p}$.

Plaut (H.-C.). - Die Hyphenpilze oder Eumyzeten im Handb. der pathog. Mikroorganismen v. Kolle u. WassermanN, 2. Aufl., Iena, 1913.

- Soor in Spez. Pathol. u. Therap. inner. Krankh. v. Kraus u. Brugsch., Berlin, 1913.

Roux (G.) et Linossien (G.). - Recherches morphologiques sur le champignon du muguet. Arch. méd. expériment. et anat. pathol., 1890, p. 5-30.

Quinguad. - Nouvelles recherches sur le muguet. Arch. de physiologie, 1868 (cité par M. Daïreuva).

Roвin (Ch.). - Des végétaux qui croissent sur l'homme et sur les animaux. Thèse de Paris, 1847.

- Histoire naturelle des végétaux parasites. Paris, 1853, p. 488-512.

Rasat (H.) et Pésu (G.). - Quelques observations sur le parasite du muguet. C. R. Soc. biol., LVIII, 1906, p. 1000-1001.

Redaelli (P.). - Cité par Grossi (G.) et Balog (P.). - Etudes cliniques et expérimentales sur la moniliase pulmonaire. Comp. rend. Cong. intern. Méd. trop. et hyg., Le Caire, 1928, II, p. 505.

ReEs. - Ueber den Soorpilz. Sitzungsb. der phys. med. Soc. zu Erlangen, 1877 (cité par M. Daïreuva).

SERgent (A.-L.). - Les facteurs de croissance des microbes sur milieux artificiels. Thèse de doct. en médecine, Paris, Doin, 1928, $182 \mathrm{p}$.

STCEGKLIN. - Recherches cliniques et expérimentales sur le rôle des levures trouvées dans les angines suspectes de diphtérie. Arch. de méd. expérim., 1898 (cité par Daïreuva).

StumpF (M.). - Untersuchungen über die Natur des Soorpilzes. Münchener Ärztl. Intelligenzblatt, 1885 , n॰ 44, p. 627, analysé dans Zentralbl.f. Klin. Med., 1886.

Talice (R.-V.). - Le facteur $p \mathrm{H}$ en mycologie. Son influence sur la culture de certaines espèces de champignons parasites de l'homme. Ann. de Parasit,. VIII, 1930, p. 183-188.

Teissier (M.). - Contribution à l'étude du champignon du muguet. Arch. de Méd. expérim. et d'anatomie pathol., 1897, n³, p. 253-276.

Section de mycologie du Laboratoire de parasitologie de la Faculté de médecine de Paris. 